\title{
Card 15 gene overexpression in mononuclear and epithelial cells of the inflamed Crohn's disease colon
}

\author{
D Berrebi, R Maudinas, J-P Hugot, M Chamaillard, F Chareyre, P De Lagausie, C Yang, \\ P Desreumaux, M Giovannini, J-P Cézard, H Zouali, D Emilie, M Peuchmaur
}

See end of article for authors' affiliations

Correspondence to: Dr D Berrebi, Service $\mathrm{d}^{\prime}$ Anatomie et de Cytologie Pathologiques, Hôpital Robert Debré, 48 bd Sérurier, 75019 Paris, France;

dominique.berrebi@ rdb.ap-hop-paris.fr

Accepted for publication 12 December 2002

\begin{abstract}
Background: Crohn's disease is one of the principal human chronic inflammatory bowel diseases. Although its aetiology is still unknown, its complex pathogenesis has environmental, immunological, and genetic determinants. CARD 15 is the first susceptibility gene implicated in the predisposition to Crohn's disease and is known to be expressed only in monocytes. However, its expression in situ has not yet been studied.

Aims: To analyse the tissue distribution of CARD 15 and identify cells producing CARD 15 in samples of colon from patients with Crohn's disease and control subjects.

Patients and methods: We analysed CARD 15 gene expression in surgical specimens of colon from eight children with Crohn's disease and nine controls by immunohistochemistry, in situ hybridisation, and reverse transcription- polymerase chain reaction (RT-PCR).

Results: We showed that CARD 15 was present only in the cytoplasm of macrophages in the normal colon. Increased CARD15 expression was detected in Crohn's disease lesions. There were more CARD 15 positive cells in Crohn's disease lesions than in uninvolved areas. Both intestinal epithelial cells, macrophages, and their derivatives overproduced CARD15 in Crohn's disease. To further assess CARD 15 expression by intestinal epithelial cells, we performed RT-PCR on freshly isolated intestinal epithelial cells, and showed that these cells isolated from Crohn's disease samples contained more CARD15 mRNA than intestinal epithelial cells from controls.

Conclusions: We have demonstrated that colonic involvement in active Crohn's disease is associated with increased CARD15 gene expression in both macrophages and intestinal epithelial cells. Therefore, this deregulation can affect the host-environment interaction and thus contribute to the pathogenesis of this disease.
\end{abstract}

Abbreviations: $C D$, Crohn's disease; CARD, caspase recruitment domain; LRR, leucin rich repeats; LPS, lipopolysaccharide; PGN, peptidoglycan; PAMP, pathogen associated molecular pattern; NFKB, nuclear factor $\kappa B$; RT-PCR, reverse transcription-polymerase chain reaction; IEC, intestinal epithelial cells; ISH, in situ hybridisation; TLR, Toll-like receptors; TNF- $\alpha$, tumour necrosis factor $\alpha$. 
Table 1 Indications for surgical treatment, presence (+) or absence (-) of granulomas, and genotype of the patient

\begin{tabular}{llll}
\hline $\begin{array}{l}\text { Patient } \\
\text { No }\end{array}$ & Indication for surgical treatment & Granuloma & Genotype \\
\hline 1 & Failure of medical treatment & - & Wild-type \\
2 & Failure of medical treatment & - & Wild-type \\
3 & Failure of medical treatment & + & Mutated heterozygote R702W \\
4 & Stenosis and fistule & + & Not done \\
5 & Failure of medical treatment & + & Mutated heterozygote 1007fs \\
6 & Failure of medical treatment & + & Wild-type \\
7 & Failure of medical treatment & + & Mutated heterozygote G908R \\
8 & Perforation & + & Compound heterozygote G908R/1007fs \\
\hline & & & \\
\end{tabular}

\section{MATERIALS AND METHODS}

\section{Surgical specimens}

Surgical specimens from involved segments of the large intestine were taken from eight paediatric patients with CD (four females and four males, mean age 14 (3.25) years). Indications for bowel resection were failure of medical treatment $(n=6)$, perforation $(n=1)$, or intestinal stenosis $(\mathrm{n}=1)$ (table 1). CD was diagnosed based on clinical, endoscopic, and histological criteria. Epithelioid and giant cell granulomas were present in six cases (table 1).The three main CD associated CARD15 mutations were genotyped, as described previously. ${ }^{18}$

Because normal colonic samples are difficult to obtain in children, controls were nine surgical specimens taken from uninvolved areas of patients with Hirschprung's disease (four females and five males, mean age 5 (4) months). Inflammatory controls were two acute appendicitis cases. Frozen tissues sections were used for immunohistochemistry and in situ hybridisation.

\section{Isolation of colon epithelial cells and peripheral blood mononuclear cells}

Epithelial cells were isolated and purified from colon specimens, as described by Toy and colleagues. ${ }^{23}$ Colon was taken from four patients with CD (two males, two females, mean age 34 (6.5) years) and five controls (four males, one female, mean age 59 (14) years) with diverticulitis $(n=3)$ or colon cancer $(n=2)$ who underwent colon resection. Mucosal layers were dissected away from the muscular and serosal layers and incubated in RPMI containing 0.5 mM DTT. Mucosal fragments were rinsed in calcium and magnesium free Hank's balanced salt solution supplemented with heat inactivated fetal calf serum (5\%), L-glutamine, and $500 \mathrm{mM}$ EDTA. Epithelial cells were collected at the interface of a Percoll gradient. The viability of epithelial cells evaluated by Trypan blue staining was greater than $85 \%$. After several washes, cells were frozen at $-20^{\circ} \mathrm{C}$. Reverse transcription-polymerase chain reaction (RT-PCR) was performed as described below. Levels of CARD15 mRNA in intestinal epithelial cells (IEC) were compared with CARD15 expression in peripheral blood mononuclear cells $\left(2 \times 10^{6} / \mathrm{ml}\right)$ separated on Ficoll gradient and collected from four of the five previous control patients.

\section{Immunohistochemistry}

Cryostat sections $(7 \mu \mathrm{m})$ of frozen tissues were cut, fixed in $100 \%$ acetone, and stored at $-20^{\circ} \mathrm{C}$. We used two rabbit polyclonal antibodies, one directed against the LRR region (a gift from Professor G Thomas, for details see immunserum below) and the other was against the CARD domain of CARD15 (Cayman Chemical, Ann Arbor, USA). Sections were washed twice in Tris $\mathrm{HCl}, \mathrm{pH}$ 7.6, and briefly in buffer containing $1 \%$ polymerised bovine albumin. They were then incubated with two blocking reagents (10\% normal human serum and $10 \%$ normal goat serum) for 30 minutes and with the primary antibodies diluted in diluent reagent (Biogenex, San Ramon, California, USA) to $1 / 1400$ (anti-LRR) and $1 / 1000$ (anti-CARD) for one hour at room temperature. CARD15 was detected using the Stravigen Multilink Kit from Biogenex, in which the multilink is biotinylated and the label is a peroxidase conjugated streptavidin. Negative controls were prepared by omitting the primary antibody and by incubation with a recombinant CARD15-LRR protein. The CARD15-LRR coding CDNA was amplified using PCR and cloned into pTrc Ha-His (Invitrogen, Rockville, USA) with SfiI- NotI. The recombinant protein (from codons 578 to 1039) containing a histidine tag was expressed in Escherichia coli and was purified using a NiNTA Superflow (Quiagen, Valencia, California, USA) column (Bio-Rad, Marnes La Coquette, France). The size and specificity of the produced recombinant protein was verified using Coomassie blue staining and anti-His western blot experiments (data not shown).

\section{Immunserum, transfection, and immunofluorescence}

CARD15 polyclonal antibody was raised in rabbits immunised with an 11 mer peptide corresponding to residues 1030KLGCRDTRLLL-1040 of CARD15 (Spi-Bio; Cayman Chemical). The entire cDNAs (approximately $3.1 \mathrm{~kb}$ pairs) encoding full length CARD15 (EMBL accession No CAC42117) was cloned under the control of the CMV promoter (Stratagene, La Jolla, California, USA). HeLa cells were plated at a density of $10^{5}$ cells/chamber on a six well plate and grown using Dulbecco's modified Eagle's medium (Life Technologies, Invitrogen) complemented with $10 \%$ fetal bovine serum, antibiotics, and glutamine. HeLa cells for immunofluorescence experiments were transfected by lipofection using DMRIE-C (Life Technologies, Invitrogen) with 500 ng of CARD15 encoding pBKCMV vector for 24 hours, accordingly to the manufacturer's instructions. Cells were fixed in 3\% paraformaldehyde, rinsed in phosphate buffered saline, permeabilised, and blocked in buffer containing $0.2 \%$ Triton X100 and $0.1 \%$ bovine serum albumin. HeLa cells were then sequentially incubated with anti-CARD15 polyclonal antibody followed by goat antirabbit secondary IgG antibodies coupled to FITC (AmershamPharmacia Biotech, Piscataway, USA). Nuclei were stained using DAPI. Slides were finally mounted onto glass coverslips using vectashield (Vector Laboratories, Burlingam, California, USA) and CARD15 located by a Zeiss Axioplan confocal fluorescence microscope.

\section{In situ hybridisation}

The digoxigenin labelled antisense riboprobe was obtained by linearising the pBK-CMV plasmid (Stratagene) containing a human CARD15 cDNA fragment (nucleotides 577-2847 of CARD15 sequence accession number: NM 022162) with EcoNl and synthesising an RNA from the T3 promoter. The digoxigenin labelled sense riboprobe was obtained by linearising the plasmid with Bam-Hl and using the T7 promoter. In situ hybridisation (ISH) experiments were performed on frozen tissue sections. ${ }^{24}$ For double labelling experiments, we 

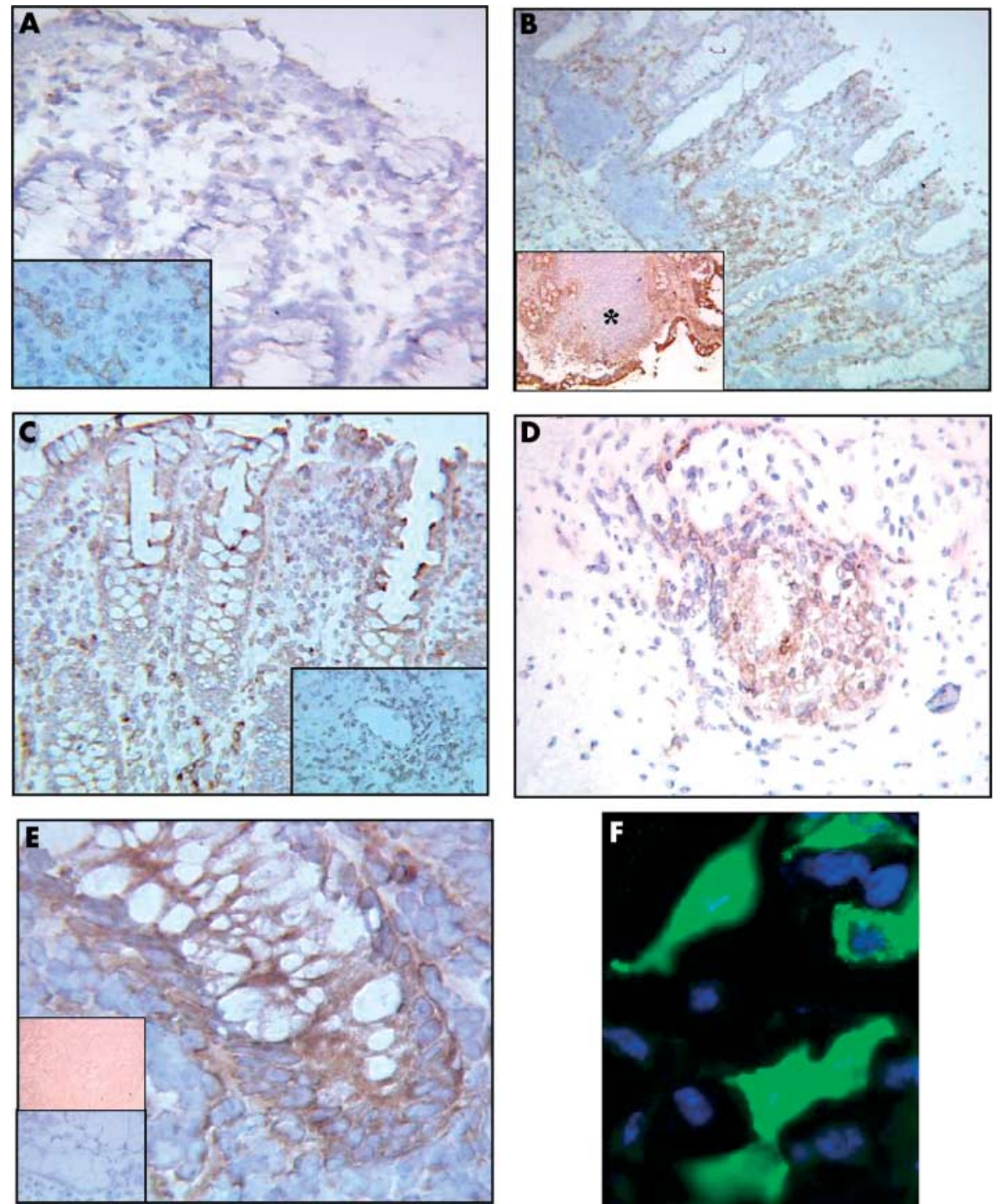

Figure 1 Immunohistochemistry using an anti-CARD 15 polyclonal antibody and frozen tissue sections of normal colon (A) and inflamed Crohn's disease colon (B-E), and subcellular distribution of CARD 15 in human cells by confocal fluorescence microscopy (F).

(A) Scattered mononuclear cells just beneath the epithelium surface, which look like macrophages (insert), are present in the normal mucosa. The Crohn's disease colon contains numerous positive cells in the lamina propria whereas no CARD 15 expression was detected within the lymphoid follicle $(B$, insert $\left({ }^{*}\right)$. Positive cells were also detected in the deeper part of the colon and around the blood vessels (C, insert). Epithelioid and giant cells in a granuloma (D) and superficial and glandular epithelial cells also produced CARD15 (B, insert; $C, E)$. ( $E$, inserts) Negative controls (using recombinant protein and primary antibody omission) showed no background staining. (F)

Nuclei were stained with DAPI

(blue). Original magnification $\times 160$ in $(B), \times 250$ in $(A), \times 400$ in $(A$, insert), (C), (D), and (E, insert), and $\times 1000$ in (E). performed ISH with the CARD15 antisense probe and immunohistochemistry with an anticytokeratin antibody directed against epithelial cells (Dako, Glostrup, Denmark) in a three stage immunoperoxidase method.

\section{Semi quantitative RT-PCR for human CARD 15}

Surgical specimens of whole colon were immediately frozen in cryotubes and stored at $-80^{\circ} \mathrm{C}$. All samples were checked before RNA extraction. Expression of $\beta$-actin and CARD15 genes was determined by RT-PCR in frozen surgical specimens and in purified IEC. Specimens were placed in RNA B (Q Biogene, Illkirch, France) according to the manufacturer's instructions in $35 \mu \mathrm{l}$ of RNA free water. DNAse enzymatic digestion was performed for 30 minutes at $37^{\circ} \mathrm{C}$ using a DNA free kit (Ambion, Austin, Texas, USA). Complementary DNA was synthesised by reverse transcription of $2 \mu \mathrm{g}$ of total RNA using SuperScript version II (Gibco BRL, Grand Island, New York, USA) and oligo(dT) ${ }_{12-18}$ primer (Gibco BRL). The negative control was the above reaction without reverse transcriptase. CARD15 was amplified using Taq polymerase (Gibco BRL) for 35 cycles under the following conditions: $95^{\circ} \mathrm{C}$ ( 40 seconds), annealing at $58^{\circ} \mathrm{C}\left(45\right.$ seconds), and extension at $72^{\circ} \mathrm{C}$ (one minute), with a final extension at $72^{\circ} \mathrm{C}$ for five minutes. PCR amplification of $\beta$-actin was conducted over 30 cycles under the following conditions: $94^{\circ} \mathrm{C}$ (one minute), $60^{\circ} \mathrm{C}$ (one minute), and $72^{\circ} \mathrm{C}$ (one min 30 seconds). The primer pairs used to amplify CARD15 were 5'-
AGCGAGACTGAGCAGACAC-3' (upstream) and 5'CAGGTTGCCGATCTTCACAC-3' (downstream) resulting in a 197 bp fragment; those for $\beta$-actin were $5^{\prime}$ GGGTCAGAAGGATTCCTATG-3' (upstream) and ${ }^{\prime}$ GGTCTCAAACATGATCTGGG-3' (downstream) resulting in a $237 \mathrm{bp}$ amplified fragment. The amplified products were subjected to gel electrophoresis and analysed by computed assisted densitometry, with NIH Image 1.62 software.

PCR products were confirmed as CARD15 by nucleotide sequencing. All sequencing was carried out using dideoxy terminator chemistry (Perkin-Elmer, Foster City, California, USA) and electrophoresed on an automated DNA sequencer.

\section{RESULTS}

\section{Immunohistochemical distribution of CARD15 in} surgically resected specimens of colon

The two CARD15 antibodies tested gave the same results. Control colon samples contained scattered positive mononuclear cells in the superficial part of the lamina propria beneath the surface epithelium (fig 1A). These positive cells possessed abundant cytoplasm, vesicular nuclei, looked like tissue macrophages, and CARD15 was detected in the cytoplasm (fig 1A, insert). Control colon samples contained no other cell types that produced CARD15. The CD colon specimens contained many mononuclear cells producing CARD15 throughout the lamina propria where they formed 

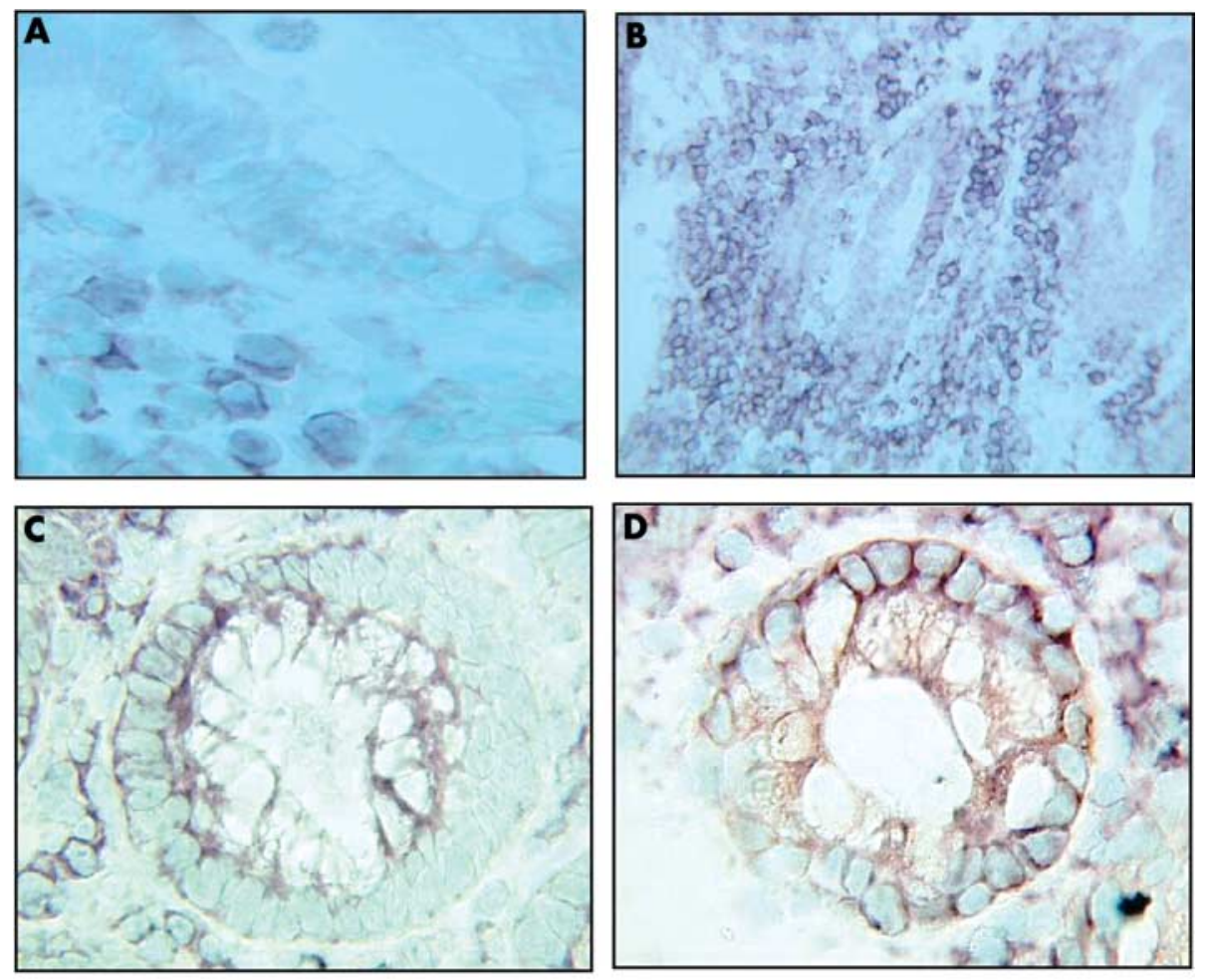

Figure 2 In situ hybridisation using a CARD15 antisense probe (A-D) and frozen tissues sections of normal colon (A) and inflamed

Crohn's disease colon (B-D).

(A) Only mononuclear cells contained CARD 15 mRNA, and no CARD 15 mRNA was detected in epithelial cells. Many cells expressing CARD 15 mRNA were present in the CD mucosa (B). Epithelial cells expressing CARD 15 mRNA (C) were identified by double labelling, detection of CARD 15 by in situ hybridisation, and epithelial cells by immunohistochemistry using an anticytokeratin antibody (D). The sense probe gave no signal $(E)$. Original magnification $\times 250$ in (B), $\times 1000$ in $(A),(C),(D)$, and $(E)$.

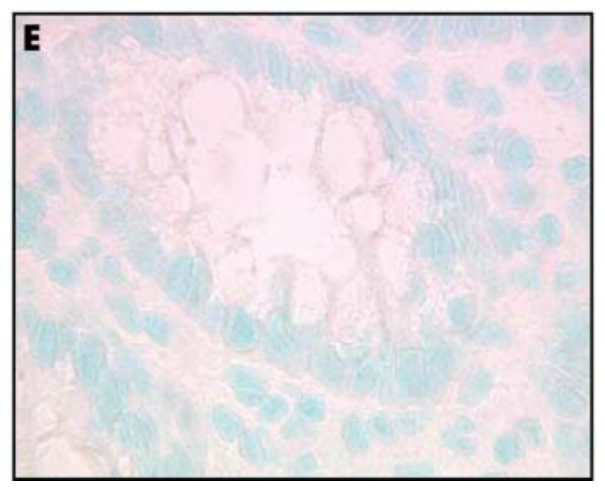

clusters (fig 1B, 1C). They were also found in the submucosa and in the deeper part of the gut, often around blood vessels (fig 1C, insert). The number of CARD15 positive cells was correlated with the intensity of the inflammatory infiltrate and was higher in areas of ulceration. There were also granulomas containing epithelioid and giant cells that immunostained for CARD15 (fig 1D). IEC also contained CARD15 in their cytoplasm, in addition to macrophages and related cells. These CARD15 positive epithelial cells were found in both the epithelial and glandular compartments in severely inflamed
CD samples (fig 1C, IE). Epithelial cells in less inflamed CD samples were less intensely immunostained, and CARD15 was only observed in a few of the glands and in only some epithelial cells within the gland. We detected no CARD15 in the lymphoid follicles or in intraepithelial lymphocytes but epithelium surrounding lymphoid follicles expressed CARD15 (fig 1B). There was no staining in any of the negative controls whether CARD15 antibody was omitted (fig IE, insert, bottom) or after preincubation with recombinant CARD15 (fig IE, insert, top).
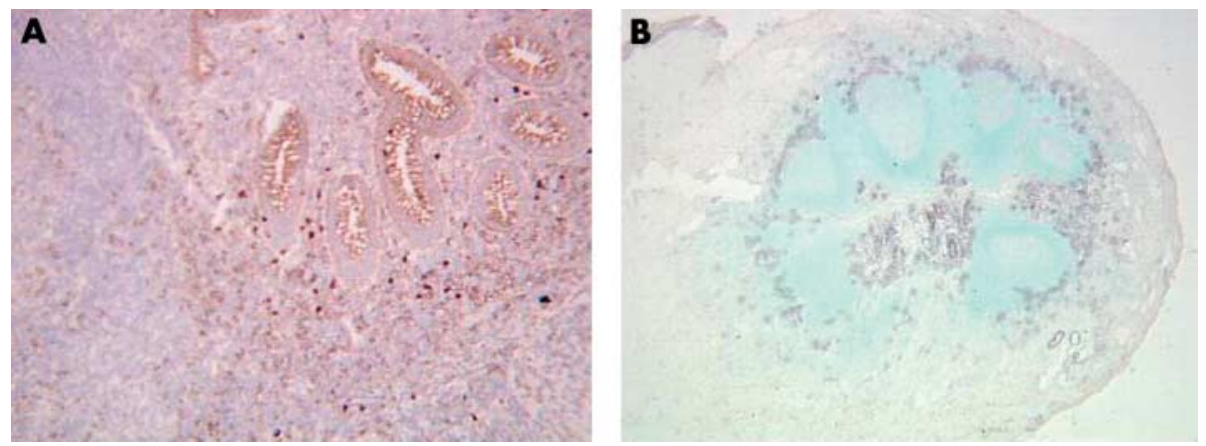

Figure 3 Immunohistochemistry using an anti-CARD 15 polyclonal antibody $(A)$ and in situ

hybridisation using a CARD 15

antisense probe $(B)$ and frozen

tissue sections from acute appendicitis cases. The inflamed appendix contains numerous positive cells in the lamina propria and in the submucosa whereas no CARD 15 expression was detected within the lymphoid follicle (B). Superficial and glandular epithelial cells also produced CARD 15

Original magnification $\times 250$ in $(A)$ and $\times 160$ in (B). 
Subcellular distribution of CARD15 in human cells By immunofluorescence and confocal microscopic examination, CARD15 was found throughout the cytoplasm, with slight punctuation (fig $\mathrm{IF}$ ), as was previously shown for CARD4/NODl. There were no large aggregates or filaments, unlike BCL-10. The profile of CARD15 distribution was confirmed with a HA tagged CARD15 construct (data not shown).

\section{Expression of CARD15 mRNA in colonic specimens In situ hybridisation (ISH) studies}

ISH confirmed the immunohistochemical data. ISH using the specific CARD15 digoxigenin labelled antisense riboprobe showed that only scattered mononuclear cells in the lamina propria of control samples contained CARD15 mRNA (fig 2A). In contrast, cells expressing CARD15 mRNA were abundant throughout the intestinal wall of CD samples (fig $2 \mathrm{~B}$ ), including granulomas (data not shown).

IEC also expressed CARD15 mRNA in CD samples (fig 2B, 2C) but no CARD15 was detected in normal IEC (fig 2A). We performed double labelling experiments to detect both CARD 15 by ISH and IEC by immunohistochemistry using an anticytokeratin antibody to demonstrate that the staining inside the glands was due to the epithelial cells and not to the mononuclear cells infiltrating the epithelium. These experiments confirmed that the IEC labelled by cytokeratin also expressed CARD 15 mRNA (fig 2D). Finally, a negative control experiment was done using a CARD15 sense riboprobe; this gave no signal (fig $2 \mathrm{E}$ ).

\section{Expression of CARD15 in acute appendicitis}

Two acute appendicitis cases were studied. Immunohistochemistry and ISH gave the same results. Numerous mononuclear cells producing CARD15 were detected in the lamina propria and in the submucosa, forming small to large clusters around the glands or around the lymphoid follicles (fig 3). In contrast, no staining was observed within the lymphoid follicles ( fig 3B). IEC also expressed CARD15 both in the surface and glandular compartments.

\section{RT-PCR studies}

Whole tissues samples

All samples were checked before mRNA extraction and the intensity of the inflammatory infiltrate as well as the presence of granulomas were noted. CARD15 mRNA was detected in all CD samples. However, the amounts of CARD15 mRNA varied from one sample to another. The amount of CARD15 mRNA was correlated with the intensity of the inflammatory infiltrate but not with the presence of granulomas. In contrast, CARD15 was detected in only four of nine normal samples. Furthermore, the odds ratio of CARD15 mRNA to control $\beta$-actin (fig 4A) was significantly higher in colon samples from CD patients than in controls (mean 207.77 in the control group $v 765$ in the CD group; unpaired Student's $t$ test, $\mathrm{p}=0.0056$ ).

Freshly isolated colon epithelial cells

To further explore CARD15 expression by IEC, we isolated colon epithelial cells from CD and control samples. By RT-PCR, CARD15 mRNA was detected in all colon epithelial cells extracted from the surgical specimens of the four patients with CD and five controls enrolled in this study (fig 4B). Levels of CARD 15 mRNA were more than five times higher in extracted epithelial cells from CD patients (632 (89), n=4) compared with controls (121 (40), $\mathrm{n}=5)$. For equivalent quantities of $\beta$-actin, similar levels of CARD15 mRNA were found in extracted normal epithelial cells compared with peripheral blood mononuclear cells (211 (22), n=4), the major cellular source of CARD15 in healthy humans (fig 4C). ${ }^{14}$

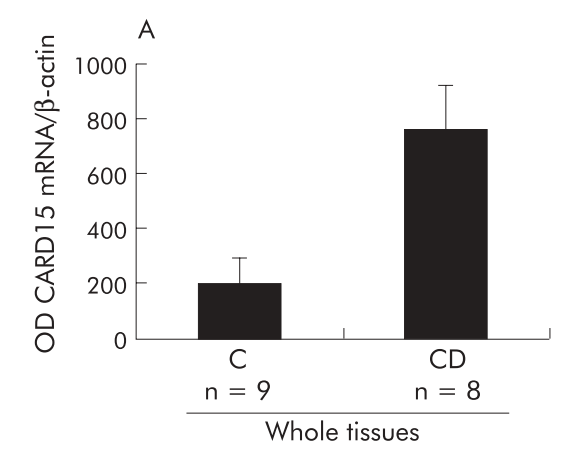

B
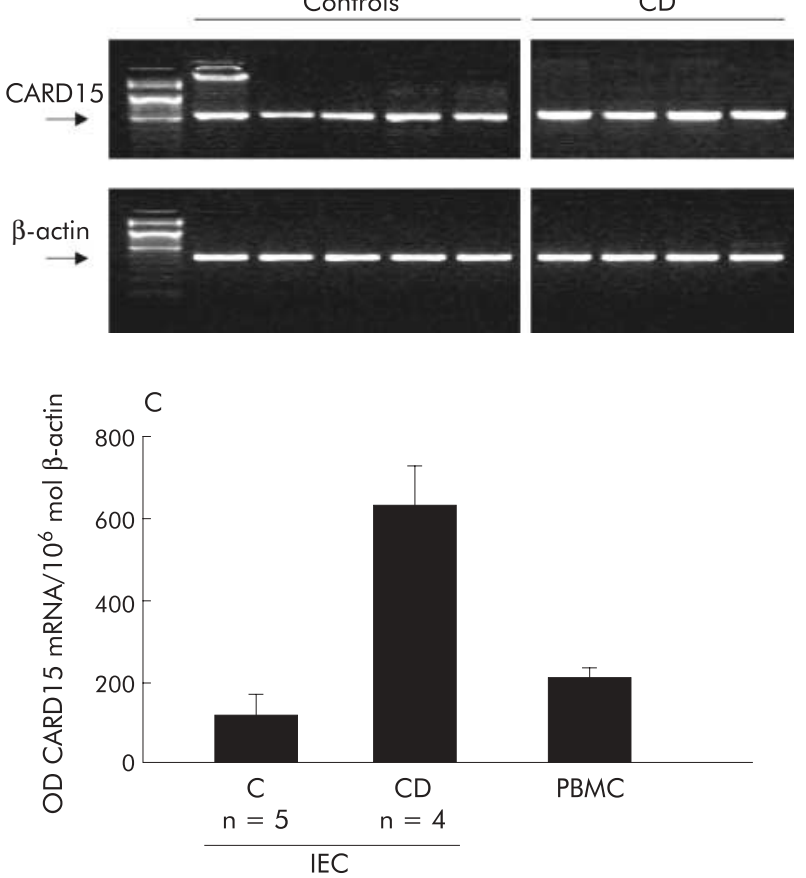

Figure 4 Reverse transcription-polymerase chain reaction (RT-PCR) analysis of CARD 15 in whole colon tissues samples (A) and in colon epithelial cells of controls (C) and Crohn's disease (CD) patients (B,

C). (A) Ratio of CARD 15 to $\beta$-actin mRNAs levels demonstrated a significant increase in whole tissue samples from CD patients compared with tissues samples from controls. Each bar represents the mean (SEM). Amplified products were subjected to gel

electrophoresis and analysed by computed assisted densitometry with NIH Image 1.62 software. (B) CARD 15 was detected in all intestinal epithelial cells extracted from surgical specimens of all patients with CD and controls enrolled in this study. (C) The level of CARD 15 mRNA was more than five times higher in extracted epithelial cells from CD patients compared with controls. For equivalent quantities of $\beta$-actin, similar levels of CARD 15 mRNA were found in extracted normal epithelial cells compared with peripheral blood mononuclear cells (PBMC). The number of patients (n) is indicated. OD, optical density.

\section{DISCUSSION}

We have investigated the intestinal expression of CARD15 in normal and CD colon and its subcellular localisation. The results of immunohistochemistry and ISH experiments were in complete agreement, and indicated that CARD15 is present in the normal colon at low levels. This observation is however consistent with previous reports based on northern blotting which failed to demonstrate reliable CARD15 expression in gut tissues ${ }^{214}$ (personal data). Using in situ techniques, we have shown that there is much more CARD15 in the inflamed colon of $\mathrm{CD}$ patients than in the normal colon where only scattered macrophages within superficial lamina propria express CARD15. The CD colon is known to contain focal 
transparietal cell infiltrates of mononuclear cells, particularly macrophages, epithelioid, and giant cells. The number of macrophages containing CARD15 was strikingly elevated throughout the CD colon wall, particularly near the blood vessels, and was correlated with the intensity of the inflammatory infiltrate. This distribution is consistent with the induced recruitment and activation of CARD15 positive macrophages by a stressful environment such as luminal bacteria in the lumen. As monocytes differentiate into macrophages and giant and epithelioid cells, leading to the formation of granulomas, we analysed expression of CARD15 in the granulomas. Epithelioid and giant cells of the granulomas both produce efficiently CARD15. Through activation of NFKB, overexpression of CARD15 could account for the NFKB dependant production of proinflammatory cytokines such as tumour necrosis factor $\alpha$ (TNF- $\alpha)$ inside the granulomas. ${ }^{25}$ Interestingly, mutations of CARD15 are found in Blau syndrome, a rare autosomal dominant disorder in which there is also a granulomatous inflammatory reaction. ${ }^{26}$ Moreover, despite a borderline significance statistical test, genotype-phenotype correlation studies in CD patients by Lesage et al primarily showed that patients with double dose mutations were associated with the presence of granulomas. ${ }^{18}$ CARD15 may thus play a pivotal role during and/or following granuloma formation in chronic inflammatory disorders.

Previous in vitro studies indicated that CARD15 gene expression was restricted to monocytes. ${ }^{214}$ This study has shown that other cell types may produce CARD 15, markedly in pathological conditions. We showed using in vitro studies that levels of CARD15 mRNA were more than five times higher in extracted epithelial cells from CD patients compared with normal colon where only very small amounts of CARD 15 were detected. Both epithelial cells on the surface and in the glandular compartment contribute to the production of CARD15 in CD. Then, IEC, the first barrier of defence against luminal bacteria, largely participate in the enhanced overall CARD15 expression in CD. In common with several CARD containing and related plant $\mathrm{R}$ (resistance) proteins, we showed that CARD15 was located in the cytoplasm, without any transmembrane position. This suggests that it may be involved in detecting ingested bacteria (that is, via PGN recognition) in antigen presenting cells. This cytoplasmic localisation is important as it has been suggested that CARDI5 is an intracytoplasmic sensor of bacterial products. ${ }^{15}$ The family of transmembrane receptors designated Toll-like receptors (TLR) are key receptors responsible for immune cell activation in response to PAMP. TLR2 and TLR4, two majors TLRs, are constitutively present at low concentrations at the apical pole of IEC. Perhaps significantly, expression of TLR- 4 is enhanced in the CD colon but that of TLR-2 (the extracellular pathogen recognition receptor of bacterial PGN) is not. ${ }^{27}$ Therefore, while the phenotype of normal IEC is apical pole TLR2 $2^{\text {low }}$ $\mathrm{CARD}^{5}{ }^{\mathrm{low}}$, CD IEC overproduce only intracellular recognition receptors for PGN, leading to the particular phenotype apical pole TLR- $2^{\text {low }} /$ cytoplasmic CARD $15^{\text {high }}$. IEC are normally refractory to extracellular LPS, which when present inside the cell, is capable of initiating an inflammatory response..$^{28}$ Commensal gut flora exist in harmony with the mucosa of the normal intestine where the epithelial phenotype is the "low responsiveness type". However, the CD intestine is no longer tolerant towards the products of lumen bacteria" ${ }^{29}$; the resulting "high responsiveness type" phenotype could lead to increased PGN recognition at the apical pole and within the cytoplasm of the epithelial cells. CARD15, similar to other members of the NOD family, interacts with RICK, a molecule that transduces signals from receptors of both innate and adaptative immune responses, allowing activation of NFKB. A recent study showed that RICK deficient cells are hyporesponsive to signalling through Nod proteins.$^{30}$ Recently, Tadakazu et al demonstrated in vitro that CARD15 was upregulated at the mRNA and protein levels by TNF- $\alpha$ (AGA abstract, 2002) in colonic epithelial cells. A more recent study showed the link between TNF- $\alpha / N F \kappa B$ and CARD15. ${ }^{31}$ These biochemical data and the observation of CARD15 expression in acute appendicitis favour the hypothesis that CARD15 may be upregulated in different inflammatory processes. CARD15 producing cells may help to protect the gut wall against aggression in normal individuals. As CARD15 is involved in innate immunity and in general inflammatory processes, its overexpression, triggered by particular PAMPs in epithelial cells, could sensitise the digestive tract and may be of major importance in the context of genetic and environmental factors allowing the development of CD. It has been reported that subjects with CARD15 gene mutations are at less risk of colon involvement and/or at greater risk of ileal disease. ${ }^{182}$ However, the observed CARD 15 overexpression in chronic inflamed colon would be explained by either CARD 15 mutations or a defect in the maintenance of an adequate CARD 15 expression. Despite the small number of patients, the first hypothesis appears to be unlikely. In contrast, modulation of CARD15 expression may thus be explained by unidentified factors, such as at least one of the identified genetic CD susceptibility loci.

This study demonstrates for the first time that CARD15 is overproduced in the $\mathrm{CD}$ colon, and is found in both macrophages and related cells and, abnormally, in mucosa epithelial cells. This dysregulated expression of the CARD15 gene may be explained by an inappropriate relationship between genetic and environmental factors. Further studies are now needed to determine whether CARD15 gene expression in $\mathrm{CD}$ and other inflammatory bowel diseases differs, and the role of luminal bacteria and/or CD susceptibility loci in induction of pathological CARD15 overexpression.

\section{ACKNOWLEDGEMENTS}

We thank Mrs Regine Paris and Mrs Agnes Florentin of the Pathology Department, Hôpital Robert Debré, for technical support.

\section{Authors' affiliations}

D Berrebi, R Maudinas, M Peuchmaur, Service d'Anatomie et de Cytologie Pathologiques, Paris, Hôpital Robert Debré, Assistance Publique-Hôpitaux de Paris France, and EA3 102, Université Paris 7 France

J-P Hugot, Service de Gastroentérologie Pédiatrique, Paris, Hôpital Robert Debré, Assistance Publique- Hôpitaux de Paris France, and EA3 102, Université Paris 7, France, and Fondation Jean Dausset-CEPH, Paris, France

M Chamaillard, F Chareyre, Fondation Jean Dausset-CEPH, Paris, France, and INSERM U434, Paris, France

P De Lagausie, Service de Chirurgie Pédiatrique, Hôpital Robert Debré, Paris, France

C Yang, P Desreumaux, Equipe Propre INSERM 0114 sur la Physiopathologie des Maladies Inflammatoires Intestinales, CHU Lille, France

M Giovannini, INSERM U434, Paris, France

J-P Cézard, Service de Gastroentérologie Pédiatrique, Paris, Hôpital Robert Debré, Assistance Publique- Hôpitaux de Paris France, and EA3102, Université Paris 7, France

H Zouali, Fondation Jean Dausset-CEPH, Paris, France

D Emilie, Inserm U131, Clamart, France

\section{REFERENCES}

1 Fiocchi C. Inflammatory bowel disease: etiology and pathogenesis. Gastroenterology 1998;115:182-205.

2 Hugot JP, Chamaillard M, Zouali $\mathrm{H}$, et al. Association of NOD2 leucine-rich repeat variants with susceptibility to Crohn's disease. Nature 2001;411:599-603

3 Ogura $Y$, Bonen DK, Inohara N, et al. A frameshift mutation in NOD2 associated with susceptibility to Crohn's disease. Nature 2001:411:603-6

4 Rioux JD, Daly M, Silverberg MS, et al. Genetic variation in the $5 q^{3} 1$ cytokine gene cluster confers susceptibility to Crohn disease. Nat Genet 2001;29:223-7.

5 Lawrance IC, Fiocchi C, Chakravarti S. Ulcerative colitis and Crohn's disease: distinctive gene expression profiles and novel susceptibility candidate genes. Hum Mol Genetics $2001 \cdot 10: 445-56$.

6 Kosiewicz MM, Nast CC, Krishnan A, et al. Th 1 -type responses mediate spontaneous ileitis in a novel murine model of Crohn's disease. J Clin Invest $2001 ; 107: 695-702$ 
7 Shanahan F. Seminar Crohn's disease. Lancet 2002;359:62-9.

8 Berrebi D, Besnard M, Fromont-Hankard G, et al . Interleukin-12 expression is focally enhanced in the gastric mucosa of pediatric patients with Crohn's disease. Am J Pathol 1998;152:667-72

9 Parrello T, Monteleone G, Cucchiara S, et al. Up-regulation of the IL-12 receptor beta 2 chain in Crohn's disease. J Immunol 2000; 165:7234-9.

10 Pizzaro TT, Michie MH, Bentz M, et al IL-18, a novel immunoregulatory cytokine, is up-regulated in Crohn's disease: expression and localization in intestinal mucosal cells. J Immunol 1999;162:6829-35.

11 Lindsay JO, Hodgson HJ. Review article: the immunoregulatory cytokine interleukin- 10-a therapy for Crohn's disease? Aliment Pharmacol Ther 2001;15:1709-16.

12 Fiocchi C. TGF-beta/Smad signaling defects in inflammatory bowel disease: mechanisms and possible novel therapies for chronic inflammation. J Clin Invest 2001;108:523-6.

13 Hugot JP, Laurent-Puig P, Gower-Rousseau C, et al. Mapping of a susceptibility locus for Crohn's disease on chromosome 16. Nature 1996; 379:821-3.

14 Ogura Y, Inohara N, Benito A, et al. Nod2, a Nod1/Apaf-1 family member that is restricted to monocytes and activates NF-KB.J Biol Chem $2001 ; 276: 4812-18$

15 Inohara N, Ogura Y, Chen FF, et al. Human Nodl confers responsiveness to bacterial lipopolysaccaharides. J Biol Chem 2001;276:2551-4.

16 Chamaillard M, Philpott D, Girardin SE, et al. Gene-environment interaction modulated by allelic heterogeneity in inflammatory diseases. Proc Natl Acad Sci USA 2003; 100:3455-60.

17 Schreiber S, Nikolaus S, Hampe J. Activation of nuclear factor $\kappa B$ in inflammatory bowel disease. Gut 1998;42:477-84.

18 Lesage S, Zouali $\mathrm{H}$, Cézard JP, et al CARD15/NOD2 mutational analysis and genotype-phenotype correlation in 612 patients with inflammatory bowel disease. Am J Hum Genet 2002;70:845-57.

19 Ahmad T, Armuzzi A, Bunce M, et al. The molecular classification of the clinical manifestations of Crohn's disease. Gastroenterology 2002 122:854-66.
20 Cuthbert AP, Fisher SA, Mirza MM, et al . The contribution of NOD2 gene mutations to the risk and site of disease in inflammatory bowel disease. Gastroenterology 2002; 122:867-74.

21 Hampe J, Grebe J, Nikolaus S, et al . Association of NOD2 (CARD15) genotype with clinical course of Crohn's disease cohort study. Lancet 2002;359:1661-5

22 Radlmayr M, Torok HP, Martin K, et al. The c-insertion mutation of the NOD2 gene is associated with fistulizing and fibrostenotic phenotypes in Crohn's disease. Gastroenterology 2002:122:2091-92.

23 Toy LS, Yio XY, Lin A, et al. Defective expression of pg 180, a novel CD8 ligand on intestinal epithelial cells in inflammatory bowel disease: J Clin Invest 1997; 100:2062-71

24 Foussat A, Bouchet-Delbos L, Berrebi D, et al. Deregulation of the expression of the fractalkine/fractalkin receptor complex in HIV-1-infected patients. Blood 2001;98:1678-86.

25 Roach DR, Bean AG, Demangel C, et al. TNF regulates chemokine induction essential for cell recruitment, granuloma formation, and clearance of mycobacterial infection. J Immunol 2002;168:4620-7.

26 Miceli-Richard C, Lesage S, Rybojad M, et al. CARD 15 mutations in Blau syndrome. Nat Genet 2001;29:19-20.

27 Cario E, Podolsky DK. Differential alteration in intestinal epithelial cell expression of Toll-Like receptor 3 (TLR3) and TLR4 in inflammatory bowel disease. Infect Immun 2000;68:7010-17.

28 Philpott DJ, Yamaoka S, Israel A, et al. Invasive Shigella flexneri activates NF-kappa B through a lipopolysaccharide-dependent innate intracellular response and leads to IL-8 expression in epithelial cells. J Immunol 2000;165:903-14.

29 Zareie M, Singh PK, Irvine EJ, et al. Monocyte/macrophage activation by normal bacteria and bacterial products. Implications for altered epithelial function in Crohn's disease. Am J Pathol 2001:158:1 101-9.

30 Kobayashi K, Inohara N, Hernandez LD, et al. RICK/Rip2/CARDIAK mediates signalling for receptors of the innate and adaptative immune system. Nature 2002;416:194-9.

31 Gutierrez O, Pipaon C, Inohara N, et al. Induction of Nod2 in myelomonocytic and intestinal epithelial cells via nuclear factor-kappa B activation. J Biol Chem 2002;277:41701-5. 